\title{
From the ICU to the operating room: how to manage the patient?
}

\author{
James Tanke/ ${ }^{\mathrm{a}, \mathrm{b}, \mathrm{c}}$, Frederic Zimmerman ${ }^{\mathrm{d}}$, Audrey De Jong ${ }^{\mathrm{e}}$ \\ Ignacio Martin-Loeches ${ }^{\mathrm{f}, \mathrm{g}}$, and Sharon Einav ${ }^{\mathrm{b}, \mathrm{d}}$
}

\begin{abstract}
Purpose of review
To outline key points for perioperative ICU optimization of nutrition, airway management, blood product preparation and transfusion, antibiotic prophylaxis and transport.
\end{abstract}

\section{Recent findings}

Optimization entails glycemic control for all, with specific attention to type-1 diabetic patients. Transportrelated adverse events may be averted with surgery in the ICU. If moving the patient is unavoidable, transport guidelines should be followed and hemodynamic optimization, airway control, and stabilization of mechanical ventilation ensured before transport. Preinduction preparation includes assessment of the airway and high-flow oxygen to prolong apneic oxygenation. Postintubation, a protective positive ventilation strategy, should be employed. Ideal transfusion thresholds are $7 \mathrm{~g} / \mathrm{dl}$ for hemodynamically stable adult patients, $8 \mathrm{~g} / \mathrm{dl}$ in orthopedic/cardiac surgery/cardiovascular disease and higher in specific disease states. Antimicrobial prophylaxis within $120 \mathrm{~min}$ of incision prevents most surgical site infections. Antibiotic therapy depends on the antibiotics being received in the ICU, the time elapsed since ICU admission, local epidemiology and the type of surgery. Tailored antimicrobial regimens may be continued periprocedurally. If more than $70 \%$ of the nutritional requirement cannot be met enterally, parenteral nutrition should be initiated within 5-7 days of surgery or earlier if the patient is malnourished.

\section{Summary}

ICU patients who require surgery may benefit from appropriate perioperative management.

\section{Keywords}

intensive care, operative, perioperative care, surgical, surgical procedures, transportation of patients

\section{INTRODUCTION}

ICU patients often require multiple surgical procedures, which may be either planned or emergent. The most complex cases are usually those that require repeat surgical interventions in the operating room because of peritonitis caused by sepsis. Complicating the care of these patients even further are the challenging decisions that must be made regarding patient preparation for transport. The following review addresses several important aspects of patient care in these specific circumstances (Fig. 1).

\section{OPTIMIZATION OF GLUCOSE CONTROL AND NUTRITION}

In surgical patients, both the antecedent illness as well as the procedure itself drive a state of catabolism and peripheral insulin resistance that can lead to abnormal glycemic homeostasis. Hyperglycemia, in particular is associated with impaired wound healing, increased length of stay, complications and mortality $[1,2]$. Similarly, hypoglycemia is

\footnotetext{
aDepartment of General Surgery, Shaare Zedek Medical Centre, ${ }^{b}$ Hebrew University Faculty of Medicine and General ICU, Jerusalem, Israel, 'Department of Surgery and Cancer, Imperial College Healthcare NHS Trust, St Mary's Hospital, London, United Kingdom, 'General Intensive Care Unit of the Shaare Zedek Medical Center, Jerusalem,

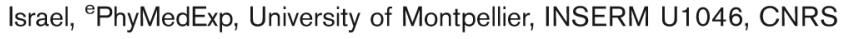
UMR, 9214, Department of Anesthesia and Intensive Care unit, Regional University Hospital of Montpellier, St-Eloi Hospital, Montpellier, France, fDepartment of Intensive Care Medicine, Multidisciplinary Intensive Care Research Organization (MICRO), St. James's Hospital, St James Street, Dublin 8, Dublin, Ireland and ${ }^{9}$ Hospital Clinic, IDIBAPS, Universidad de Barcelona, Ciberes, Barcelona, Spain

Correspondence to Professor Sharon Einav, MSc, MD, Director, Surgical Intensive Care Unit, Shaare Zedek Medical Centre, Affiliated with the Hebrew University, POB 3235, Jerusalem 91031, Israel.

Tel: +972 2 6666664; fax: +972 2 6555144;

e-mail: einav_s@szmc.org.il
} 
- Adequate preparation for surgery in ICU patients requires nutritional optimization, careful airway management, appropriate preparation of blood products, antibiotic prophylaxis and carefully planned patient transport.

- Serum glycemia should be maintained between 140 and $180 \mathrm{mg} / \mathrm{dl}$ with continuous insulin infusions in type 1 diabetics to avoid ketoacidosis.

- Enteral nutrition should ideally be withheld during planned intervention and reinstated shortly thereafter.

- If more than $70 \%$ of the nutritional requirement cannot be met enterally, parenteral nutrition should be initiated within 5-7 days of surgery and earlier in the setting of malnourishment.

- Unstable patients with severe hypoxemia require airway control and stabilization with mechanical ventilation before transport.

- Hemodynamic stabilization may require intravenous fluids and vasopressors before induction of anesthesia.

- During rapid sequence induction, high oxygen flows maintains apneic oxygenation.

- Following intubation, tube placement should be verified with capnography and a protective positive ventilation strategy employed.

- A transfusion threshold of $7 \mathrm{~g} / \mathrm{dl}$ is recommended for hemodynamically stable adult patients, with $8 \mathrm{~g} / \mathrm{dl}$ recommended in orthopedic or cardiac surgery and in patients with cardiovascular disease.

- Preoperative cross matching of packed red blood cells is only necessary for procedures with a risk of significant and rapid hemorrhage.

- Timely antimicrobial prophylaxis (within 120-min of incision) may prevent $80 \%$ of surgical site infections.

- Patients who are receiving no antibiotics in the ICU and those taken from the ICU to the operating room less than $48 \mathrm{~h}$ following ICU admission should receive preprocedural prophylactic antibiotics in accordance with standard recommendations.

- Patients in the ICU for more than $48 \mathrm{~h}$ who are already being treated with a tailored antimicrobial regimen may continue this regimen periprocedurally.

- Bedside surgery in the ICU decreases transport-related adverse events.

- When moving the patient is unavoidable, implementation of transport protocols may avert transport-related complications. associated with adverse neurological sequalae [3] and increased morbidity in critically ill patients [4].

Preoperative optimization of glycemic control ideally starts before the date of surgery as an elevated HbA1c is independently associated with perioperative complications [5]. Contrary to elective surgery, which usually requires a $20-30 \%$ dose reduction in insulin for fasting diabetics regardless of type [6,7], the ICU patient is likely to arrive to the operating room fasting with or without a short-acting insulin drip. Critically ill patients are typically hyperglycemic because of an ongoing stress response, and are therefore often managed in the ICU with a shortacting insulin infusion. Although this infusion may or may not have been withheld for the sake of simplifying patient transfer, it must be restarted in the operating room for fasting patients with type 1 diabetes in order to avoid ketoacidosis [8"']. Outside of emergency surgery, oral and noninsulin injectable antihyperglycemic medications should be stopped on the morning of surgery [9].

During surgery, and in the immediate postoperative period, blood glucose monitoring is required at 1-2 hourly intervals. Early recommendations to maintain blood glucose at less than $110 \mathrm{mg} / \mathrm{dl}$ [10] were found less efficacious in avoiding morbidity compared with a more conservative target of 140$180 \mathrm{mg} / \mathrm{dl}$ [11]. If the patient remains hyperglycemic despite an insulin infusion, they should be treated with a bolus of intravenous insulin and an increase in the baseline intravenous dose. Hypoglycemia should be treated with intravenous 5\% dextrose. Serum potassium levels should be monitored alongside serum glucose especially when exogenous insulin is administered.

Following surgery, once enteral feeding is reinstituted, basal insulin can be restarted although a dose reduction may be needed if caloric intake is poor. When replacing intravenous with subcutaneous insulin, a $2 \mathrm{~h}$ overlap between the two modes of administration should be ensured. With the exception of Metformin, which should only be restarted with adequate enteral hydration, oral antihyperglycemic medications may be restarted once enteral diet has been successfully established. Metformin should be restarted only once adequate hydration is also achieved.

Serum albumin poorly reflects nutritional status during critical illness. Although nutritional optimization ideally starts at least 7-10 days preoperatively, this is often not possible in most ICU patients. Malnourishment in the ICU is common and associated either with premorbid state or acute inflammation. When the stress of acute illness occurs in the setting of malnutrition, lean body muscle decreases precipitously [12]. As malnutrition 
in the ICU is associated with infectious complications [13] and mortality in emergency surgical patients [14], preventing nutritional decline is paramount.

Nutritional support should be considered for all patients requiring greater than $48 \mathrm{~h}$ of ICU admission as admissions exceeding this time-frame have an increased risk of malnutrition [15]. Continuous enteral nutrition may be withheld during the period of surgery itself and ideally reinstituted in the ICU immediately after surgery. Nutritional requirements should be met gradually over $48 \mathrm{~h}$ and overnutrition avoided [15]. If more than $70 \%$ of the nutritional requirement cannot be met with enteral feeding, or if enteral feeding is contraindicated, supplementation via an alternative route is needed [16]. Parenteral nutrition should be initiated within 5-7 days, although if the patient is clinically or biochemically malnourished before surgery, parenteral nutrition should be initiated earlier.

\section{MANAGEMENT OF THE AIRWAY AND VENTILATION}

Management of patients with borderline hypoxemia and complicated noninvasive or invasive ventilation is challenging. Therefore, oxygenation and ventilatory support of critically ill patients should ideally be provided in a well monitored and specialist-led ICU. Optimal support of those patients requiring surgery is particularly difficult for both the intensivist and the anesthesiologist; adverse events related to airway management and mechanical ventilation are more likely to occur in ICU patients requiring treatment in the operating room than in patients undergoing elective surgery regardless of the location of intubation [17]. To reduce the risk of adverse events in critically ill patients [18"], the following strategies should be implemented.

If the patient is being treated in the ICU with noninvasive positive pressure ventilation, transport may be unsafe if a high flow nasal cannula or high flow oxygen of more than $15 \mathrm{l} / \mathrm{min}$ is required to maintain an oxygen saturation of more than $90 \%$. In this situation, gas exchange may deteriorate during transport. Furthermore, as urgent airway management in suboptimal conditions has been associated with a very high risk of complications [19-21], airway control and stabilization with mechanical ventilation should occur before transport to the operating room.

Before proceeding to intubation, the predicted difficulty should be assessed using scores specifically developed for critically ill patients, such as the MACOCHA score [22-23]. Severe dyspnea is often accompanied by aerophagia with resultant gastric distension, which puts the patient at increased risk of aspiration even when fasted. Therefore, in the setting of critical illness, rapid sequence induction is the technique of choice to secure the patient's airway [24]. Beforehand, preoxygenation should be optimized with noninvasive ventilation [25]. Following induction, apneic oxygenation must be maintained by using high flow oxygen via the nares [26"]. The Montpellier Intubation Protocol, which was developed to prevent complications associated with airway management in ICU patients, can easily be adapted for intubation of critically ill patients in the operating room. In addition to preoxygenation and rapid sequence induction, this protocol includes hemodynamic optimization with fluid loading and vasopressors prior to induction of anesthesia and verification of correct tube placement with capnography [24].

Following intubation, a protective positive ventilation strategy should be used including low tidal volumes, moderate to high positive end-expiratory pressures (PEEP) and recruitment maneuvers [27]. Manual insufflations without monitoring of inspiratory pressures should be avoided [28]. Although esophageal pressure monitoring may help to titrate PEEP [29], PEEP should be balanced in order to prevent atelectases whilst simultaneously avoiding over-distension and hemodynamic compromise [30]. Spontaneous breathing has been associated with earlier weaning from mechanical ventilation and discharge from the ICU, and should therefore be allowed if possible [31]. Fastidious monitoring with revision of ventilator settings to limit lung injury will prevent the development or worsening of the acute respiratory distress syndrome.

\section{BLOOD PRODUCT PREPARATION AND TRANSFUSION}

Although 10 years ago, the practice of regular blood tests was ostracized for critically ill patients [32-34], many still undergo multiple daily blood sampling. Preoperative anemia has been associated with 30-day postoperative mortality, acute myocardial infarction, acute ischemic stroke, central nervous system complications and acute kidney injury [35"'].

As the association between perioperative anemia is thought to arise from the presence of underlying chronic or severe disease rather than from the anemia itself, preoperative transfusion is not key to preventing postoperative complications. In the setting of orthopedic [36] and cardiac surgery [37,38], sepsis [39], major burns [40], upper gastrointestinal hemorrhage [41], a restrictive transfusion trigger (hemoglobin of $7-8 \mathrm{~g} / \mathrm{dl}$ ) is similar to a liberal transfusion trigger (hemoglobin $<10 \mathrm{~g} / \mathrm{dl}$ ) in terms 
of short-term outcomes. Restrictive transfusion triggers also do not affect quality of life [42] or the rate of rehabilitation from physical disability [43] after surgery.

Current international guidelines strongly recommend a restrictive transfusion threshold for all hospitalized adult patients who are hemodynamically stable. A somewhat higher but still restrictive transfusion threshold of $8 \mathrm{~g} / \mathrm{dl}$ is used for patients undergoing orthopedic or cardiac surgery and those with preexisting (i.e. chronic) cardiovascular disease. However, those patients with an acute coronary syndrome, those with bleeding diathesis who are at risk of bleeding and patients who have chronic transfusion-dependent anemia should have their hemoglobin maintained above $10 \mathrm{~g} / \mathrm{dl}$ [44"].

Wasting of blood products is an important quality measure as high wastage reflects poor use of an important resource. Measurements of the efficiency of packed red blood cell usage (pRBC), such as the ratio of $\mathrm{pRBC}$ units cross matched to those transfused (C:T ratio), packed red blood cell unit wastage rates, and $\mathrm{RBC}$ unit expiration rates have been determined in large hospital datasets. Ideally, a C:T ratio below 2 , pRBC unit expiration rates below $1.0 \%$, and pRBC unit wastage rate below $0.5 \%$ can be achieved [45]. In order to achieve these targets, cross matching of pRBC should only be performed for those patients at risk of clinically significant rapid and hemorrhage.

\section{ANTIBIOTIC ADMINISTRATION}

Over two-thirds of critically ill patients receive antibiotics during their ICU stay [46]. ICU patients are particularly susceptible to both infectious complications and drug-related side-effects. Patient comorbidities and treatment-related factors, such as the presence of intravascular catheters, artificial airways and life support devices contribute to the former. In contrast, the presence of multiorgan failure, increased creatinine clearance and volume of distribution contribute to the latter. The resultant changes in the pharmacokinetic/pharmacodynamic (PK/PD) of antibiotics may render them more likely to accumulate or, alternatively, to be ineffectual because of low therapeutic levels.

Antimicrobial prophylaxis may prevent up to $80 \%$ of surgical site infections (SSI) [47] especially in the setting of contaminated surgery and specific patient subgroups such as those with diabetes [48]. In order for prophylactic treatment to be effective it must also be administered in a timely fashion with guidelines recommending within a $120-\mathrm{min}$ prior to the skin incision [49"-]. Antibiotic prophylaxis regimens vary dependent on the clinical setting, the expected surgical procedure, patient characteristics and local infectious disease epidemiology including patterns of antibiotic resistance. Further to these considerations are adjustments of antibiotic dosage and treatment duration following surgery in order to optimize treatment [49"'].

The antibiotic coverage provided to critically ill patients is often broad-spectrum. Although this strategy is considered the best way to prevent the development of multidrug-resistant (MDR) pathogens, repeated exposure to broad spectrum antibiotics may conversely create an environment for MDR pathogens to develop. Nevertheless, in the setting of antibiotics used for surgical prophylaxis, there is no association with an increased risk of antibiotic-resistant postoperative infections [50].

Whilst the guidelines for antimicrobial prophylaxis in routine operating room cases are supported by a high level of evidence [51"], the level of evidence regarding prophylaxis in critically ill patients taken from the ICU to the operating room is much lower. A recent retrospective observational study suggested that for those patients admitted to the ICU more than $48 \mathrm{~h}$ before surgery, the antimicrobial therapy given in the ICU could be continued in the operating room [52]. In those patients admitted to the ICU less than $48 \mathrm{~h}$ before their surgical procedure, antimicrobial therapy should be given in accordance with standard preoperative recommendations. Antibiotic prophylaxis should cover the local flora, taking into consideration the specific surgery at hand and patient-related factors that may affect drug pharmacokinetics/pharmacodynamics.

A national, propensity-score-adjusted, retrospective, cohort study, which compared antibiotic prophylaxis with either vancomycin with a betalactam versus the use of either drug alone for prevention of SSI found that specifically in cardiac surgery, combination prophylaxis was associated with a reduction in SSIs. However, this was also associated with an increase in postoperative acute kidney injury [53].

\section{Transport}

The movement of patients to the operating room constitute between 15 and 50\% of all intra-hospital transports (IHTs) [54]. Early studies demonstrated a $5 \%$ immediate mortality associated with adverse events during IHT $[55,56]$. However, whilst IHT itself has not been associated with excess mortality [21,57], it has been associated with poor longer term outcomes $[21,54,57,58]$. Transport from the operating room to the ICU, as opposed to transport from the ICU, is particularly susceptible to adverse events $[56,59]$.

Adverse events may be reduced by reducing IHT. Thus, before exposing the critically ill patient to the 
risks of transport, a decision must be made with the surgical team regarding the balance between potentially suboptimal surgery in the ICU versus IHT to the operating room with the associated risks of transport. For this reason, many procedures previously performed in the operating room are now being performed in the ICU. This includes the most commonly performed bedside procedure, tracheostomy insertion $[60,61]$, as well as abdominal closure [62], diagnostic laparoscopy [63] and neurosurgical external ventricular drain placement [64]. Although no randomized studies have compared patient outcomes following ICU versus operating room surgical procedures, some such procedures are considered to be well tolerated and have outcomes at least equivalent to those procedures performed in the operating room $[60,63]$. It is important to note, however, that evidence does vary with regards to these outcomes [64].

In situations that IHT is unavoidable, the risks of IHT must be mitigated where possible. Those patients at the highest risk of complications during transport are patients who are ventilated $[21,55,65,66]$, patients with central nervous system injury [67] and patients that are hemodynamically unstable [21,59]. Major adverse events associated with IHT include cardiovascular (arrhythmias, hypotension and hypertension), respiratory (desaturation, obstruction or dislodgment of endotracheal tube) and neurological sequalae (elevated intracranial pressure), equipment malfunction (loss of battery power or oxygen supply) and human factors (inappropriate mechanical ventilation settings, transportation of the wrong patient) $[21,58,59,65,68,69]$. Any immediately nonessential devices, such as hemofiltration, or medication, such as diuretics, should be discontinued for the duration of IHT in order to simplify the transfer process. The equipment used for transport and the equipment available in the operating room may not have settings similar to those used in the ICU. If this situation is anticipated, a trial using modified equipment settings to emulate the conditions that will be available either during transport or in the operating room should be undertaken in the ICU before moving the patient. If the patient remains stable, IHT may be undertaken immediately. However, if deterioration is noted, alternative solutions may need to be sought.

As the complications associated with IHT were first described in the early 1970s [55], significant strides have been made in optimizing IHT. Guidelines recommending interventions to reduce complications related to transport have been produced and supported by various professional societies [66,70-73]. Checklists have been developed to aid the implementation of these protocols with a view to further reduce complications $[21,59,65,74]$. These guidelines and checklists focus, among other things,

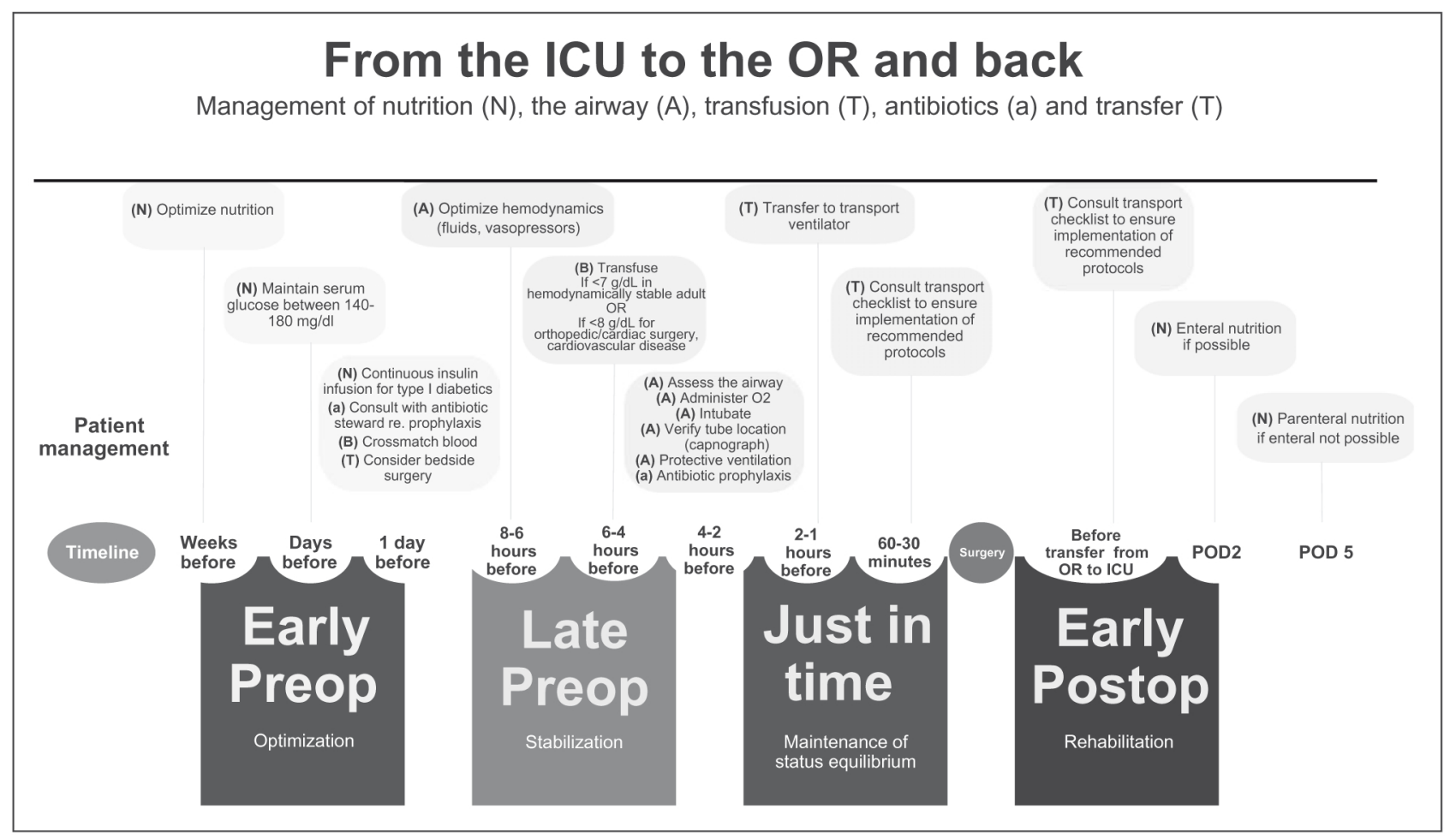

FIGURE 1. Timeline of patients management for critically ill patients requiring surgery. The aspects of care covered include nutrition, the airway, transfusion, antibiotics and transport. 
on physician and critical care nurse participation in the transport team, adequate training of transport teams, sufficient pretransport preparation, effective and reliable equipment and communication within the transport team and between the transferring and receiving departments [59].

\section{CONCLUSION}

Adequate preparation of ICU patients for surgery requires an awareness of the elements that ultimately affect patient outcome. In this review, some, but not all, of the important aspects of care for these patients are discussed. Perioperative optimization of nutrition, airway management, blood product preparation, antibiotic prophylaxis and transport have all been associated with the outcomes of critically ill surgical patients who need to be transported around the hospital, and thus merit special attention when the question of additional surgery is raised in these inherently complex patients.

\section{Acknowledgements}

Author contributions: All the authors collected information and wrote sections of the manuscript. J.T. and S.E. merged the information and edited the text to create the final version of the manuscript. The manuscript in its final form was approved by all of the authors.

\section{Financial support and sponsorship}

None.

\section{Conflicts of interest}

J.T. and F.Z. report no conflicts of interest. A.D.J., personal fees from Baxter and Medtronic-Covidien, and travel reimbursements from Fresenius-Kabi, MSD France, Astellas, Pfizer and Fisher Paykel. I.M.L., personal fees from MSD and Gilead.

S.E., patents with Medtechnica, consulting for MSD and Medtechnica, travel cost reimbursements from Zoll, Medtechnica and Diasorin, lecture for Fisher \& Paykel with no travel cost reimbursements or financial renumeration, participation in multicentre trials run by Artisanpharma, Eisai and Astra Zeneca.

\section{REFERENCES AND RECOMMENDED READING}

Papers of particular interest, published within the annual period of review, have been highlighted as:

- of special interest

-1. of outstanding interest

1. Frisch A, Chandra P, Smiley D, et al. Prevalence and clinical outcome of hyperglycemia in the perioperative period in noncardiac surgery. Diabetes Care 2010; 33:1783-1788.

2. Umpierrez GE, Isaacs SD, Bazargan N, et al. Hyperglycemia: An independent marker of in-hospital mortality in patients with undiagnosed diabetes. J Clin Endocrinol Metab 2002; 87:978-982.
3. Soldevila B, Lucas AM, Zavala R, Mauricio D. Chapter 8: Perioperative management of the diabetic patient. In: perioperative medicine - current controversies. Ed: Karen Stuart-Smith; Springer International Publishing 2016. ISBN 973-3-319-28819-2.

4. Investigators TN-SS. Intensive versus conventional glucose control in critically ill patients. N Engl J Med 2009; 360:1283-1297.

5. Jehan F, Khan M, Sakran JV, et al. Perioperative glycemic control and postoperative complications in patients undergoing emergency general surgery: what is the role of plasma hemoglobin A1c? J Trauma Acute Care Surg 2018; 84:112-117.

6. Joshi GP, Chung F, Vann MA, et al., Society for Ambulatory Anesthesia. Society for ambulatory anesthesia consensus statement on perioperative blood glucose management in diabetic patients undergoing ambulatory surgery. Anesth Analg 2010; 111:1378-1387.

7. Clement S, Braithwaite SS, Magee MF, et al., American Diabetes Association Diabetes in Hospitals Writing Committee. Management of diabetes and hyperglycemia in hospitals. Diabetes Care 2004; 27:553-591.

8. Leung V, Ragbir-Toolsie K. Perioperative management of patients with

I. diabetes. Health Serv Insights 2017; 10: 1178632917735075. doi: $10.1177 / 1178632917735075$.

An up-to-date and concise discussion of the key elements of managing diabetic patients throughout the perioperative period.

9. Umpierrez GE, Hellman R, Korytkowski MT, et al. Management of hyperglycemia in hospitalized patients in noncritical care setting: an endocrine society clinical practice guideline. J Clin Endocrinol Metab 2012; 97:16-38.

10. Den Berghe V, Wouters $P$, Weekers $F$, et al. Intensive insulin therapy in critically ill patients. New Engl J Med J 2001

11. Tanner $O$. Intensive versus conventional glucose control in critically ill patients. https://journals.sagepub.com/doi/abs/10.1177/175114370901000314.

12. Cederholm $T$, Bosaeus $I$, Barazzoni $R$, et al. Diagnostic criteria for malnutrition An ESPEN Consensus Statement. Clin Nutr 2015; 34:335-340.

13. Rubinson $L$, Diette GB, Song $X$, et al. Low caloric intake is associated with nosocomial bloodstream infections in patients in the medical intensive care unit. Crit Care Med 2004; 32:350-357.

14. Havens JM, Columbus $A B$, Seshadri AJ, et al. Malnutrition at intensive care unit admission predicts mortality in emergency general surgery patients. $J$ Parenter Enter Nutr 2016; 42:156-163.

15. Singer $P$, Berger MM, Van den Berghe $G$, et al. ESPEN guidelines on parenteral nutrition: intensive care. Clin Nutr 2009; 28:387-400.

16. McClave SA, Taylor BE, Martindale RG, et al., Society of Critical Care Medicine; American Society for Parenteral and Enteral Nutrition. Guidelines for the provision and assessment of nutrition support therapy in the adult critically ill patient: Society of Critical Care Medicine (SCCM) and American Society for Parenteral and Enteral Nutrition (A.S.P.E.N.). J Parenter Enter Nutr 2016; 40:159-211.

17. De Jong $A$, Molinari $N$, Pouzeratte $Y$, et al. Difficult intubation in obese patients: incidence, risk factors, and complications in the operating theatre and in intensive care units. $\mathrm{Br} J$ Anaesth 2015; 114:297-306.

18. De Jong A, Rolle A, Molinari N, et al. Cardiac arrest and mortality related to

- intubation procedure in critically ill adult patients: a multicenter cohort study. Crit Care Med 2018; 46:532-539.

Multicenter study showing that intubation-related cardiac arrest occurred in $1: 40$ ICU intubations and was independently associated with mortality. The main risk factors were obesity, age greater than 75 years and preintubation systolic hypotension, hypoxemia or lack of preoxygenation.

19. Parmentier-Decrucq E, Poissy J, Favory R, et al. Adverse events during intrahospital transport of critically ill patients: incidence and risk factors. Ann Intensive Care 2013; 3:10.

20. Veiga VC, Postalli NF, Alvarisa TK, et al. Adverse events during intrahospital transport of critically ill patients in a large hospital. Rev Bras Ter Intensiva 2019; 31:15-20.

21. Fanara $B$, Manzon $C$, Barbot $O$, et al. Recommendations for the intra-hospital transport of critically ill patients. Crit Care 2010; 14:R87.

22. De Jong A, Molinari N, Terzi N, et al. Early identification of patients at risk for difficult intubation in ICU: development and validation of the MACOCHA Score in a Multicenter Cohort Study. Am J Respir Crit Care Med 2013; $187: 832-839$

23. De Jong $A$, Jung $B$, Jaber $S$. Intubation in the ICU: we could improve our practice. Crit Care 2014; 18:209.

24. Jaber $S$, Jung $B$, Corne $P$, et al. An intervention to decrease complications related to endotracheal intubation in the intensive care unit: a prospective, multiple-center study. Intensive Care Med 2010; 36:248-255.

25. Frat JP, Ricard JD, Quenot JP, et al. Noninvasive ventilation versus high-flow nasal cannula oxygen therapy with apnoeic oxygenation for preoxygenation before intubation of patients with acute hypoxaemic respiratory failure: a randomised, multicentre, open-label trial. Lancet Respir Med 2019; 7:303-312.

26. Jaber $S$, Monnin $M$, Girard $M$, et al. Apnoeic oxygenation via high-flow nasal

- cannula oxygen combined with noninvasive ventilation preoxygenation for intubation in hypoxaemic patients in the intensive care unit: the single-centre, blinded, randomised controlled OPTINIV trial. Intensive Care Med 2016; 42:1877-1887. 
Proof of concept study suggesting that, in patients with severe hypoxemic respiratory failure, preoxygenation with HFNC and NIV together is more effective in preventing oxygen desaturation than with NIV alone.

27. Futier $\mathrm{E}$, Constantin JM, Paugam-Burtz C, et al. A trial of intraoperative lowtidal-volume ventilation in abdominal surgery. N Engl J Med 2013; 369:428-437.

28. Ball L, Hemmes SNT, Serpa Neto A, et al., LAS VEGAS investigators; PROVE Network; Clinical Trial Network of the European Society of Anaesthesiology. Intraoperative ventilation settings and their associations with postoperative pulmonary complications in obese patients. $\mathrm{Br} J$ Anaesth 2018; 121:899-908.

29. Pereira SM, Tucci MR, Morais CCA, et al. Individual positive end-expiratory pressure settings optimize intraoperative mechanical ventilation and reduce postoperative atelectasis. Anesthesiology 2018; 129:1070-1081.

30. Eichler L, Truskowska K, Dupree A, et al. Intraoperative ventilation of morbidly obese patients guided by transpulmonary pressure. Obes Surg 2018; 28:122-129.

31. Van Haren F, Pham T, Brochard L, et al., Large observational study to UNderstand the Global impact of Severe Acute respiratory FailurE (LUNG SAFE) Investigators. Spontaneous breathing in early acute respiratory distress syndrome: insights from the Large Observational Study to UNderstand the Global Impact of Severe Acute Respiratory FailurE Study. Crit Care Med $2019 ; 47: 229-238$

32. Thavendiranathan $P$, Bagai $A$, Ebidia $A$, et al. Do blood tests cause anemia in hospitalized patients? The effect of diagnostic phlebotomy on hemoglobin and hematocrit levels. J Gen Intern Med 2005; 20:520.

33. Ranasinghe T, Freeman WD. ICU vampirism' - time for judicious blood draws in critically ill patients. Br J Haematol 2014; 164:302-303.

34. Chant C, Wilson G, Friedrich JO. Anemia, transfusion, and phlebotomy practices in critically ill patients with prolonged ICU length of stay: a cohort study. Crit Care 2006; 10:R140.

35. Mueller MM, Van Remoortel $H$, Meybohm $P$, et al., ICC PBM Frankfurt 2018

n. Group. Patient blood management: recommendations from the 2018 Frankfurt Consensus Conference. JAMA 2019; 321:983-997.

A thorough summary of the existing literature on patient blood management including metanalyses of specific questions where the data lends itself to such analyses.

36. Carson JL, Terrin ML, Noveck $\mathrm{H}$, et al., FOCUS Investigators. Liberal or restrictive transfusion in high-risk patients after hip surgery. N Engl J Med 2011; 365:2453-2462.

37. Murphy GJ, Pike K, Rogers CA, et al., TITRe2 Investigators. Liberal or restrictive transfusion after cardiac surgery. N Engl J Med 2015; 372:997-1008.

38. Mazer CD, Whitlock RP, Fergusson DA, et al., TRICS Investigators and Perioperative Anesthesia Clinical Trials Group. Restrictive or liberal red-cell transfusion for cardiac surgery. N Engl J Med 2017; 377:2133-2144.

39. Holst LB, Haase N, Wetterslev J, et al., TRISS Trial Group; Scandinavian Critical Care Trials Group. Lower versus higher hemoglobin threshold for transfusion in septic shock. N Engl J Med 2014; 371:1381-1391.

40. Palmieri TL, Holmes $\mathrm{JH} 4$ th, Arnoldo $\mathrm{B}$, et al. Transfusion requirement in burn care evaluation (TRIBE): a multicenter randomized prospective trial of blood transfusion in major burn injury. Ann Surg 2017; 266:595-602.

41. Villanueva $C$, Colomo $A$, Bosch $A$, et al. Transfusion strategies for acute upper gastrointestinal bleeding. N Engl J Med 2013; 368:11-21.

42. Gregersen M, Borris LC, Damsgaard EM. Blood transfusion and overall quality of life after hip fracture in frail elderly patients-the transfusion requirements in frail elderly randomized controlled trial. J Am Med Dir Assoc 2015; 16:762-766.

43. Gregersen M, Borris LC, Damsgaard EM. Postoperative blood transfusion strategy in frail, anemic elderly patients with hip fracture: the TRIFE randomized controlled trial. Acta Orthop 2015; 86:363-372.

44. Carson JL, Guyatt $\mathrm{G}$, Heddle NM, et al. Clinical practice guidelines from the

- AABB: red blood cell transfusion thresholds and storage. JAMA 2016; 316:2025-2035.

Practical transfusion guidelines based on data and multidisciplinary expert opinion.

45. Novis DA, Renner S, Friedberg R, et al. Quality indicators of blood utilization: three College of American Pathologists Q-Probes studies of 12,288,404 red blood cell units in 1639 hospitals. Arch Pathol Lab Med 2002; 126:150-156

46. Vincent JL, Rello J, Marshall J, et al., EPIC II Group of Investigators. International study of the prevalence and outcomes of infection in intensive care units. JAMA 2009; 302:2323-2329.

47. Berrios-Torres SI, Umscheid CA, Bratzler DW, et al., Healthcare Infection Control Practices Advisory Committee. Centers for Disease Control and Prevention Guideline for the Prevention of Surgical Site Infection, 2017. JAMA Surg 2017; 152:784-791.

48. Righy C, do Brasil PEA, Vallés J, et al. Systemic antibiotics for preventing ventilator-associated pneumonia in comatose patients: a systematic review and meta-analysis. Ann Intensive Care 2017; 7:67.

49. de Jonge SW, Gans SL, Atema JJ, et al. Timing of preoperative antibiotic

- prophylaxis in 54,552 patients and the risk of surgical site infection: a systematic review and meta-analysis. Medicine (Baltimore) 2017; 96:e6903.

A systematic review and meta-analysis that supports administration of prophylactic antibiotics within the 120-min time frame prior to incision. The broadly accepted recommendation to administer prophylaxis within a 60-min time frame prior to incision could not be substantiated.

50. Cohen ME, Salmasian H, Li J, et al. Surgical antibiotic prophylaxis and risk for postoperative antibiotic-resistant infections. J Am Coll Surg 2017; 225:631.e3-638.e3.

51. Martin C, Auboyer $C$, Boisson $M$, et al., Steering committee of the French

- Society of Anaesthesia and Intensive Care Medicine (SFAR) responsible for the establishment of the guidelines.. Antibioprophylaxis in surgery and interventional medicine (adult patients). Update 2017. Anaesth Crit Care Pain Med 2019; pii: S2352-5568(19)30085-2. doi: 10.1016/j.accpm.2019.02.017. [Epub ahead of print]

Excellent review of the factors and interventions affecting the immune defences.

52. Duclos G, Pastene B, Depeyre F, et al. Surgical antimicrobial prophylaxis in intensive care unit (ICU) patients: a preliminary, observational, retrospective study. Ann Transl Med 2018; 6:402.

53. Branch-Elliman W, Ripollone JE, O'Brien WJ, et al. Risk of surgical site infection, acute kidney injury, and Clostridium difficile infection following antibiotic prophylaxis with vancomycin plus a beta-lactam versus either drug alone: a national propensity-score-adjusted retrospective cohort study. PLoS Med 2017; 14:e1002340.

54. Voigt LP, Pastores SM, Raoof ND, et al. Review of a large clinical series: intrahospital transport of critically ill patients: outcomes, timing, and patterns. J Intensive Care Med 2009; 24:108-115.

55. Waddell G. Movement of criticall ill patients within hospital. Br Med J 1975; 2:417-419.

56. Insel J, Weissman $\mathrm{C}$, Kemper $\mathrm{M}$, et al. Cardiovascular changes during transport of critically ill and postoperative patients. Crit Care Med 1986; 14:539-542.

57. Szem JW, Hydo LJ, Fischer E, et al. High-risk intrahospital transport of critically ill patients: safety and outcome of the necessary 'road trip'. Crit Care Med 1995; 23:1660-1666.

58. Waydhas C. Equipment review: Intrahospital transport of critically ill patients. Crit Care 1999; 3:R83-R89.

59. Nakayama DK, Lester SS, Rich DR, et al. Quality improvement and patient care checklists in intrahospital transfers involving pediatric surgery patients. J Pediatr Surg 2012; 47:112-118.

60. Kornblith LZ, Burlew CC, Moore EE, et al. One thousand bedside percutaneous tracheostomies in the surgical intensive care unit: time to change the gold standard. J Am Coll Surg 2011; 212:163-170.

61. Kluge $\mathrm{S}$, Baumann $\mathrm{HJ}$, Maier $\mathrm{C}$, et al. Tracheostomy in the intensive care unit: a nationwide survey. Anesth Analg 2008; 107:1639-1643.

62. Diaz JJ, Mejia V, Subhawong AP, et al. Protocol for bedside laparotomy in trauma and emergency general surgery: a low return to the operating room. Am Surg 2005; 71:986-991.

63. Alemanno G, Prosperi $P$, Di Bella A, et al. Bedside diagnostic laparoscopy for critically ill patients in the intensive care unit: Retrospective study and review of literature. J Minim Access Surg 2019; 15:56-62.

64. Foreman PM, Hendrix P, Griessenauer CJ, et al. External ventricular drain placement in the intensive care unit versus operating room: evaluation of complications and accuracy. Clin Neurol Neurosurg 2015; 128:94-100.

65. Beckmann U, Gillies D, Berenholtz S, et al. Incidents relating to the intrahospital transfer of critically ill patients. Intensive Care Med 2004; 30:1579-1585

66. Warren J, Fromm RE, Orr RA, et al., American College of Critical Care Medicine. Guidelines for the inter- and intrahospital transport of critically ill patients. Crit Care Med 2004; 32:256-262.

67. Picetti E, Antonini MV, Lucchetti MC, et al. Intra-hospital transport of braininjured patients: a prospective, observational study. Neurocrit Care 2013; 18:298-304.

68. Damm C, Vandelet $\mathrm{P}$, Petit J, et al. Complications during the intrahospital transport in critically ill patients. Ann Fr Anesth Reanim 2005; 24:24-30.

69. Papson JP, Russell KL, Taylor DM. Unexpected events during the intrahospital transport of critically ill patients. Acad Emerg Med 2007; 14:574-577.

70. Quenot J-P, Milési C, Cravoisy A, et al. Intrahospital transport of critically ill patients (excluding newborns) recommendations of the Société de Réanimation de Langue Française (SRLF), the Société Française d'Anesthésie et de Réanimation (SFAR), and the Société Française de Médecine d'Urgence (SFMU). Ann Intensive Care 2012; 2:1.

71. Guidelines for transport of critically ill patients. Australasian College for Emergency Medicine (ACEM), Australian and New Zealand College of Anaesthetists (ANZCA), College of Intensive Care Medicine of Australia and New Zealand (CICM). Available at: http://www.anzca.edu.au/documents/ps52-2015-guidelines-for-transport-of-critically-i [Accessed 2 June 2019]

72. SIAARTI Study Group for Safety in Anesthesia and Intensive Care. Recommendations on the transport of critically ill patient. Minerva Anestesiol 2006; $72: ;$ XXXVII-LVII.

73. Ferdinande $P$. Recommendations for intra-hospital transport of the severely head injured patient. Working Group on Neurosurgical Intensive Care of the European Society of Intensive Care Medicine. Intensive Care Med 1999; 25:1441-1443.

74. Brunsveld-Reinders AH, Arbous MS, Kuiper SG, de Jonge E. A comprehensive method to develop a checklist to increase safety of intra-hospital transport of critically ill patients. Crit Care 2015; 19:214. 